\title{
On the regions containing all the zeros of polynomials and related analytic functions
}

\author{
N. A. Rather, I. Dar, A. Iqbal \\ University of Kashmir, Srinagar, 190006, India
}

For citation: Rather N. A., Dar I., Iqbal A. On the regions containing all the zeros of polynomials and related analytic functions. Vestnik of Saint Petersburg University. Mathematics. Mechanics. Astronomy, 2021, vol. 8 (66), issue 2, pp. 331-337.

https://doi.org/10.21638/spbu01.2021.212

In this paper, by using standard techniques we shall obtain results with relaxed hypothesis which give zero bounds for the larger class of polynomials. Our results not only generalizes several well-known results but also provide better information about the location of zeros. We also obtain a similar result for analytic functions. In addition to this, we show by examples that our result gives better information on the zero bounds of polynomials than some known results.

Keywords: polynomials, zeros, complex domain.

1. Introduction and statement of results. Various experimental observations and investigations when translated into mathematical language lead to mathematical models. The solution of these models could lead to problems of solving algebraic polynomial equations of certain degree. The exact computation of zeros of polynomials of degree at most four made possible by virtue of algorithms having been devised for such polynomials, no such method is available for accomplishing the same task for polynomials of higher degree. The impossibility of achieving this feat, or in other words, the impossibility of solving by radicals the polynomial equations of degree 5 or greater is an important milestone in the history of mathematics, occasioned by ground breaking discoveries in algebra by N.H. Abel and E. Galois in the first quarter of the nineteenth century. In view of this and significant applications of zero bounds in scientific disciplines such as stability theory, mathematical biology, communication theory and computer engineering, it became interesting to identify the suitable regions in the complex plane which contain the zeros of a given polynomial. A classical result due to Cauchy [1] on the distribution of zeros of a polynomial may be stated as follows:

Theorem A. Let $P(z)=z^{n}+a_{n-1} z^{n-1}+\cdots+a_{1} z+a_{0}$ be a polynomial of degree $n$, then all the zeros of $P(z)$ lie in the disk $|z| \leq 1+\max _{0 \leq j \leq n-1}\left|a_{j}\right|$.

Although various results concerning the bounds for zeros of polynomials are available in literature [2], but the remarkable property of the bound in Theorem A which distinguishes it from other such bounds is its simplicity of computations. However, this simplicity comes at the cost of precision. The following elegant result on the location of zeros of a polynomial with restricted coefficients is known as Eneström - Kakeya Theorem (see $[2,3]$ ) which states that:

(c) St. Petersburg State University, 2021 
Theorem B. Let $P(z)=a_{n} z^{n}+a_{n-1} z^{n-1}+\cdots+a_{1} z+a_{0}$ be a polynomial of degree $n$ with real coefficients satisfying $a_{n} \geq a_{n-1} \geq \cdots \geq a_{1} \geq a_{0} \geq 0$, then all the zeros of $P(z)$ lie in $|z| \leq 1$.

Joyal, Labelle and Rahman [4] extended Theorem B to polynomials whose coefficients are monotonic but are not necessarily non-negative and proved that:

Theorem C. Let $P(z)=a_{n} z^{n}+a_{n-1} z^{n-1}+\cdots+a_{1} z+a_{0}$ be a polynomial of degree $n$ with real coefficients satisfying $a_{n} \geq a_{n-1} \geq \cdots \geq a_{1} \geq a_{0}$, then all the zeros of $P(z)$ lie in $|z| \leq \frac{a_{n}-a_{0}+\left|a_{0}\right|}{\left|a_{n}\right|}$.

Aziz and Zargar [5] relaxed the hypothesis in several ways and among other things they proved the following results:

Theorem D. Let $P(z)=a_{n} z^{n}+a_{n-1} z^{n-1}+\cdots+a_{1} z+a_{0}$ be a polynomial of degree $n$ with real coefficients such that for some $k \geq 1, k a_{n} \geq a_{n-1} \geq \cdots \geq a_{1} \geq a_{0}$, then all the zeros of $P(z)$ lie in $|z+k-1| \leq \frac{k a_{n}-a_{0}+\left|a_{0}\right|}{\left|a_{n}\right|}$.

In literature there exist several generalisations of above results (for reference see $[2,3]$ and [6]). In this paper, we have obtained results with relaxed hypothesis which give zero bounds for the larger class of polynomials as compared to the above discussed Eneström - Kakeya type results. In addition to this, we also obtained similar kind of results of analytic functions. Our results not only generalizes several well-known results but also provide better information about the location of zeros. We begin by proving the following result:

Theorem 1. Let $P(z)=\sum_{j=0}^{n} a_{j} z^{j}, a_{j}=\alpha_{j}+i \gamma_{j}$ be a polynomial of degree $n$ with complex coefficients such that for some $k_{j} \geq 1, \alpha_{n-j+1}>0, j=1,2, \ldots, r$ where $1 \leq r \leq n$,

$$
k_{1} \alpha_{n} \geq k_{2} \alpha_{n-1} \geq k_{3} \alpha_{n-2} \geq \cdots \geq k_{r} \alpha_{n-r+1} \geq \alpha_{n-r} \geq \cdots \geq \alpha_{1} \geq \alpha_{0},
$$

then all the zeros of $P(z)$ lie in

$$
\begin{aligned}
& \left|z+\left(k_{1}-1\right) \frac{\alpha_{n}}{a_{n}}-\left(k_{2}-1\right) \frac{\alpha_{n-1}}{a_{n}}\right| \leq \\
& \leq \frac{1}{\left|a_{n}\right|}\left[k_{1} \alpha_{n}-\left(k_{2}-1\right)\left|\alpha_{n-1}\right|+2\left(\sum_{j=2}^{r}\left(k_{j}-1\right)\left|\alpha_{n-j+1}\right|+\sum_{j=0}^{n-1}\left|\gamma_{j}\right|\right)-\alpha_{0}+\left|\alpha_{0}\right|+\left|\gamma_{n}\right|\right] .
\end{aligned}
$$

If all the coefficients of $P(z)$ are real then the above theorem reduces to the following result:

Corollary 1. Let $P(z)=a_{n} z^{n}+a_{n-1} z^{n-1}+\cdots+a_{1} z+a_{0}$ be a polynomial of degree $n$ with real coefficients such that for some $k_{j} \geq 1, \alpha_{n-j+1}>0, j=1,2, \ldots, r$ where $1 \leq r \leq n$,

$$
k_{1} a_{n} \geq k_{2} a_{n-1} \geq k_{3} a_{n-2} \geq \cdots \geq k_{r} a_{n-r+1} \geq a_{n-r} \geq \cdots \geq a_{1} \geq a_{0},
$$

then all the zeros of $P(z)$ lie in

$\left|z+k_{1}-1-\left(k_{2}-1\right) a_{n-1} / a_{n}\right| \leq \frac{1}{\left|a_{n}\right|}\left(k_{1} a_{n}-\left(k_{2}-1\right)\left|a_{n-1}\right|+2 \sum_{j=2}^{r}\left(k_{j}-1\right)\left|a_{n-j+1}\right|-a_{0}+\left|a_{0}\right|\right)$. 
Remark. For $r=2$ and $k_{2}=1$, Corollary 1 reduces to Theorem D and if $k_{j}=$ $1, j=1,2, \ldots, r$, then Corollary 1 reduces to Theorem C.

If we assume $a_{0} \geq 0$ in Corollary 1 , we get the following result:

Corollary 2. Let $P(z)=a_{n} z^{n}+a_{n-1} z^{n-1}+\cdots+a_{1} z+a_{0}$ be a polynomial of degree $n$ with real coefficients such that for some $k_{j} \geq 1, j=1,2, \ldots, r$ where $1 \leq r \leq n$,

$$
k_{1} a_{n} \geq k_{2} a_{n-1} \geq k_{3} a_{n-2} \geq \cdots \geq k_{r} a_{n-r+1} \geq a_{n-r} \geq \cdots \geq a_{1} \geq a_{0} \geq 0,
$$

then all the zeros of $P(z)$ lie in

$$
\left|z+k_{1}-1-\left(k_{2}-1\right) a_{n-1} / a_{n}\right| \leq \frac{1}{a_{n}}\left(k_{1} a_{n}-\left(k_{2}-1\right) a_{n-1}+2 \sum_{j=2}^{r}\left(k_{j}-1\right) a_{n-j+1}\right) .
$$

Now we turn to the study of the zeros of a class of analytic functions. In this direction, we prove the following result:

Theorem 2. Let $f(z)=\sum_{j=0}^{\infty} a_{j} z^{j} \not \equiv 0$ be analytic in $|z| \leq t$. If for some $k_{1}, k_{2} \geq 1$,

$$
k_{1} a_{0} \geq k_{2} t a_{1} \geq t^{2} a_{2} \geq t^{3} a_{3} \geq \ldots, \quad \text { where } a_{j}>0, j=0,1,2,3 \ldots,
$$

then $f(z)$ does not vanish in the region

$$
\left|z-\frac{\left(k_{1}-1\right) a_{0} t-\left(k_{2}-1\right) a_{1} t^{2}}{\left(2 k_{1}-1\right)\left(a_{0}+2\left(k_{2}-1\right) a_{1} t\right)}\right|<\frac{k_{1} a_{0} t+\left(k_{2}-1\right) a_{1} t^{2}}{\left(2 k_{1}-1\right)\left(a_{0}+2\left(k_{2}-1\right) a_{1} t\right)} .
$$

For $k_{2}=1$, Theorem 2 yields the following result of Aziz and Shah [7]:

Corollary 3. Let $f(z)=\sum_{j=0}^{\infty} a_{j} z^{j} \neq \equiv 0$ be analytic in $|z| \leq t$. If for some $k \geq 1$,

$$
k a_{0} \geq t a_{1} \geq t^{2} a_{2} \geq t^{3} a_{3} \geq \ldots, \quad \text { where } a_{j}>0, j=0,1,2,3 \ldots,
$$

then $f(z)$ does not vanish in the region

$$
\left|z-\frac{(k-1) t}{2 k-1}\right|<\frac{k t}{2 k-1}
$$

For $t=1$, Theorem 2 reduces to the following result:

Corollary 4. Let $f(z)=\sum_{j=0}^{\infty} a_{j} z^{j} \not \equiv 0$ be analytic in $|z| \leq 1$. If for some $k_{1}, k_{2} \geq 1$,

$$
k_{1} a_{0} \geq k_{2} a_{1} \geq a_{2} \geq a_{3} \geq \ldots \quad \text { where } a_{j}>0, j=0,1,2,3 \ldots,
$$

then $f(z)$ does not vanish in the region

$$
\left|z-\frac{\left(k_{1}-1\right) a_{0}-\left(k_{2}-1\right) a_{1}}{\left(2 k_{1}-1\right)\left(a_{0}+2\left(k_{2}-1\right) a_{1}\right)}\right|<\frac{k_{1} a_{0}+\left(k_{2}-1\right) a_{1}}{\left(2 k_{1}-1\right)\left(a_{0}+2\left(k_{2}-1\right) a_{1}\right)} .
$$


2. Computations. In this section, we give some examples of polynomials to show that Theorem 1 gives better information about the location of the zeros than Theorem A. It is worth mentioning that all existing Eneström - Kakeya type results are not applicable for these polynomials.

Example 1. Let $P(z)=3 z^{8}+2.8 z^{7}+2.9 z^{6}+3.1 z^{5}+3.2 z^{4}+3 z^{3}+2.6 z^{2}+2 z+1$. By taking $r=4$ with $k_{1}=16 / 15, k_{2}=8 / 7, k_{3}=32 / 29$ and $k_{4}=32 / 31$ in Theorem 1 , it follows that all the zeros of $P(z)$ lie in the disc $|z-1 / 15| \leq 1.4667$. Whereas, if we use Theorem A, it follows that all the zeros of $P(z)$ lie in the disc $|z|<2.0667$. Thus, Theorem 1 gives better bound, with $50 \%$ improvement in the area over Theorem A.

Example 2. Let $P(z)=19 z^{4}+18 z^{3}+20 z^{2}+15 z+2$. By taking $r=2$ with $k_{1}=20 / 19$ and $k_{2}=10 / 9$ in Theorem 1 , it follows that all the zeros of $P(z)$ lie in the disc $|z-0.52| \leq 1.157$. Whereas, if we use Theorem A, it follows that all the zeros of $P(z)$ lie in the disc $|z|<2.053$. Thus, Theorem 1 gives better bound, with $68 \%$ improvement in the area over Theorem A.

\section{Proof of the Theorems.}

Proof of Theorem 1. Consider the polynomial

$$
\begin{aligned}
& \quad F(z)=(1-z) P(z)= \\
& =-a_{n} z^{n+1}+\left(\alpha_{n}-\alpha_{n-1}\right) z^{n}+\left(\alpha_{n-1}-\alpha_{n-2}\right) z^{n-1}+\cdots+\left(\alpha_{n-r}-\alpha_{n-r-1}\right) z^{n-r}+\cdots+ \\
& +\left(\alpha_{1}-\alpha_{0}\right) z+\alpha_{0}+i\left[\left(\gamma_{n}-\gamma_{n-1}\right) z^{n}+\left(\gamma_{n-1}-\gamma_{n-2}\right) z^{n-1}+\cdots+\left(\gamma_{1}-\gamma_{0}\right) z+\gamma_{0}\right]= \\
& \quad=-a_{n} z^{n+1}+\left(k_{1} \alpha_{n}-k_{2} \alpha_{n-1}-\left(k_{1}-1\right) \alpha_{n}+\left(k_{2}-1\right) \alpha_{n-1}\right) z^{n}+ \\
& \quad+\left(k_{2} \alpha_{n-1}-k_{3} \alpha_{n-2}-\left(k_{2}-1\right) \alpha_{n-1}+\left(k_{3}-1\right) \alpha_{n-2}\right) z^{n-1}+\cdots+ \\
& \quad+\left(k_{r-1} \alpha_{n-r+2}-k_{r} \alpha_{n-r+1}-\left(k_{r-1}-1\right) \alpha_{n-r+2}+\left(k_{r}-1\right) \alpha_{n-r+1}\right) z^{n-r+2}+ \\
& +\left(k_{r} \alpha_{n-r+1}-\alpha_{n-r}-\left(k_{r}-1\right) \alpha_{n-r+1}\right) z^{n-r+1}+\left(\alpha_{n-r}-\alpha_{n-r-1}\right) z^{n-r}+\cdots+\left(\alpha_{1}-\alpha_{0}\right) z+\alpha_{0}+ \\
& \quad+i\left[\left(\gamma_{n}-\gamma_{n-1}\right) z^{n}+\left(\gamma_{n-1}-\gamma_{n-2}\right) z^{n-1}+\cdots+\left(\gamma_{1}-\gamma_{0}\right) z+\gamma_{0}\right],
\end{aligned}
$$

which implies,

$$
\begin{gathered}
|F(z)|=\mid-a_{n} z^{n+1}-\left(k_{1}-1\right) \alpha_{n} z^{n}+\left(k_{1} \alpha_{n}-k_{2} \alpha_{n-1}\right) z^{n}+\left(k_{2}-1\right) \alpha_{n-1} z^{n}+ \\
+\left(k_{2} \alpha_{n-1}-k_{3} \alpha_{n-2}\right) z^{n-1}-\left(k_{2}-1\right) \alpha_{n-1} z^{n-1}+\left(k_{3}-1\right) \alpha_{n-2} z^{n-1}+\cdots+ \\
+\left(k_{r-1} \alpha_{n-r+2}-k_{r} \alpha_{n-r+1}\right) z^{n-r+2}- \\
-\left(k_{r-1}-1\right) \alpha_{n-r+2} z^{n-r+2}+\left(k_{r}-1\right) \alpha_{n-r+1} z^{n-r+2}+\left(k_{r} \alpha_{n-r+1}-\alpha_{n-r}\right) z^{n-r+1}- \\
-\left(k_{r}-1\right) \alpha_{n-r+1} z^{n-r+1}+\left(\alpha_{n-r}-\alpha_{n-r-1}\right) z^{n-r}+\cdots+\left(\alpha_{1}-\alpha_{0}\right) z+\alpha_{0}+ \\
+i\left[\left(\gamma_{n}-\gamma_{n-1}\right) z^{n}+\left(\gamma_{n-1}-\gamma_{n-2}\right) z^{n-1}+\cdots+\left(\gamma_{1}-\gamma_{0}\right) z+\gamma_{0}\right] \mid,
\end{gathered}
$$

that is,

$$
\begin{aligned}
& |F(z)| \geq|z|^{n}\left[\left|z a_{n}+\left(k_{1}-1\right) \alpha_{n}-\left(k_{2}-1\right) \alpha_{n-1}\right|-\right. \\
& \quad-\left(\left|k_{1} \alpha_{n}-k_{2} \alpha_{n-1}\right|+\left|k_{2} \alpha_{n-1}-k_{3} \alpha_{n-2}\right| /|z|+\right. \\
& +\left|k_{2}-1\right|\left|\alpha_{n-1}\right| /|z|+\left|k_{3}-1\right|\left|\alpha_{n-2}\right| /|z|+\cdots+\left|k_{r-1} \alpha_{n-r+2}-k_{r} \alpha_{n-r+1}\right| /|z|^{r-2}+ \\
& +\left|k_{r-1}-1\right|\left|\alpha_{n-r+2}\right| /|z|^{r-2}+\left|k_{r}-1\right|\left|\alpha_{n-r+1}\right| /|z|^{r-2}+\left|k_{r} \alpha_{n-r+1}-\alpha_{n-r}\right| /|z|^{r-1}+
\end{aligned}
$$




$$
\begin{array}{r}
+\left|k_{r}-1\right|\left|\alpha_{n-r+1}\right| /|z|^{r-1}+\left|\alpha_{n-r}-\alpha_{n-r-1}\right| /|z|^{r}+\cdots+\left|\alpha_{1}-\alpha_{0}\right| /|z|^{n-1}+\left|\alpha_{0}\right| /|z|^{n}+ \\
\left.\left.+\left|\gamma_{n}-\gamma_{n-1}\right|+\left|\gamma_{n-1}-\gamma_{n-2}\right| /|z|+\cdots+\left|\gamma_{1}-\gamma_{0}\right| /|z|^{n-1}+\left|\gamma_{0}\right| /|z|^{n}\right)\right] .
\end{array}
$$

By using hypothesis, we have for $|z|>1$,

$$
\begin{aligned}
|F(z)| \geq|z|^{n}\left[\left|z a_{n}+\left(k_{1}-1\right) \alpha_{n}-\left(k_{2}-1\right) \alpha_{n-1}\right|-\left(k_{1} \alpha_{n}-k_{2} \alpha_{n-1}+k_{2} \alpha_{n-1}-k_{3} \alpha_{n-2}+\right.\right. \\
+\left(k_{2}-1\right)\left|\alpha_{n-1}\right|+\left(k_{3}-1\right)\left|\alpha_{n-2}\right|+\cdots+k_{r-1} \alpha_{n-r+2}-k_{r} \alpha_{n-r+1}+ \\
\quad+\left(k_{r-1}-1\right)\left|\alpha_{n-r+2}\right|+\left(k_{r}-1\right)\left|\alpha_{n-r+1}\right|+k_{r} \alpha_{n-r+1}-\alpha_{n-r}+ \\
\quad+\left(k_{r}-1\right)\left|\alpha_{n-r+1}\right|+\alpha_{n-r}-\alpha_{n-r-1}+\cdots+\alpha_{1}-\alpha_{0}+\left|\alpha_{0}\right|+ \\
\left.\left.\quad+\left|\gamma_{n}\right|+\left|\gamma_{n-1}\right|+\left|\gamma_{n-1}\right|+\left|\gamma_{n-2}\right|+\cdots+\left|\gamma_{1}\right|+\left|\gamma_{0}\right|+\left|\gamma_{0}\right|\right)\right],
\end{aligned}
$$

implies,

$$
\begin{aligned}
|F(z)| \geq\left|a_{n}\right||z|^{n}[\mid z & +\left(k_{1}-1\right) \frac{\alpha_{n}}{a_{n}}-\left(k_{2}-1\right) \frac{\alpha_{n-1}}{a_{n}} \mid-\frac{1}{\left|a_{n}\right|}\left\{k_{1} \alpha_{n}-\left(k_{2}-1\right)\left|\alpha_{n-1}\right|+\right. \\
& \left.\left.+2\left(\sum_{j=2}^{r}\left(k_{j}-1\right)\left|\alpha_{n-j+1}\right|+\sum_{j=0}^{n-1}\left|\gamma_{j}\right|\right)-\alpha_{0}+\left|\alpha_{0}\right|+\left|\gamma_{n}\right|\right\}\right]>0
\end{aligned}
$$

if

$$
\begin{aligned}
\mid z+\left(k_{1}-1\right) \frac{\alpha_{n}}{a_{n}}-\left(k_{2}-1\right) & \frac{\alpha_{n-1}}{a_{n}} \mid>\frac{1}{\left|a_{n}\right|}\left[k_{1} \alpha_{n}-\left(k_{2}-1\right)\left|\alpha_{n-1}\right|+\right. \\
& \left.+2\left(\sum_{j=2}^{r}\left(k_{j}-1\right)\left|\alpha_{n-j+1}\right|+\sum_{j=0}^{n-1}\left|\gamma_{j}\right|\right)-\alpha_{0}+\left|\alpha_{0}\right|+\left|\gamma_{n}\right|\right] .
\end{aligned}
$$

This shows that those zeros of $F(z)$ whose modulus is greater than 1 lie in

$$
\begin{aligned}
\mid z+\left(k_{1}-1\right) \frac{\alpha_{n}}{a_{n}}-\left(k_{2}-1\right) & \frac{\alpha_{n-1}}{a_{n}} \mid \leq \frac{1}{\left|a_{n}\right|}\left[k_{1} \alpha_{n}-\left(k_{2}-1\right)\left|\alpha_{n-1}\right|+\right. \\
& \left.+2\left(\sum_{j=2}^{r}\left(k_{j}-1\right)\left|\alpha_{n-j+1}\right|+\sum_{j=0}^{n-1}\left|\gamma_{j}\right|\right)-\alpha_{0}+\left|\alpha_{0}\right|+\left|\gamma_{n}\right|\right] .
\end{aligned}
$$

But those zeros of $F(z)$ whose modulus is less than or equal to 1 already lie in this region. Hence it follows that all the zeros of $F(z)$ and therefore of $P(z)$ lie in

$$
\begin{aligned}
\mid z+\left(k_{1}-1\right) \frac{\alpha_{n}}{a_{n}}-\left(k_{2}-1\right) & \frac{\alpha_{n-1}}{a_{n}} \mid \leq \frac{1}{\left|a_{n}\right|}\left[k_{1} \alpha_{n}-\left(k_{2}-1\right)\left|\alpha_{n-1}\right|+\right. \\
& \left.+2\left(\sum_{j=2}^{r}\left(k_{j}-1\right)\left|\alpha_{n-j+1}\right|+\sum_{j=0}^{n-1}\left|\gamma_{j}\right|\right)-\alpha_{0}+\left|\alpha_{0}\right|+\left|\gamma_{n}\right|\right] .
\end{aligned}
$$

This completes the proof of Theorem 1. 
Proof of Theorem 2. Since $f(z)=\sum_{j=0}^{\infty} a_{j} z^{j}$ is analytic in $|z| \leq t$ and it is easy to observe that $\lim _{j \rightarrow \infty} t^{j} a_{j}=0$. Now consider the function

$$
\begin{gathered}
F(z)=(z-t) f(z)=-t a_{0}+\left(a_{0}-t a_{1}\right) z+\left(a_{1}-t a_{2}\right) z^{2}+\left(a_{2}-t a_{3}\right) z^{3}+\cdots= \\
=-t a_{0}+\left(k_{1} a_{0}-t k_{2} a_{1}\right) z-\left(\left(k_{1}-1\right) a_{0}-\left(k_{2}-1\right) t a_{1}\right) z+ \\
+\left(\left(k_{2} a_{1}-t a_{2}\right)-\left(k_{2}-1\right) a_{1}\right) z^{2}+\left(a_{2}-t a_{3}\right) z^{3}+\cdots=-t a_{0}-\left(\left(k_{1}-1\right) a_{0}-\left(k_{2}-1\right) t a_{1}\right) z+\phi(z),
\end{gathered}
$$

where $\phi(z)=\left(k_{1} a_{0}-t k_{2} a_{1}\right) z+\left(\left(k_{2} a_{1}-t a_{2}\right)-\left(k_{2}-1\right) a_{1}\right) z^{2}+\sum_{j=3}^{\infty}\left(a_{j-1}-t a_{j}\right) z^{j}$.

Clearly $\phi(z)$ is analytic for $|z| \leq t$ with $\phi(0)=0$. Moreover, for $|z|=t$

$$
\begin{array}{r}
|\phi(z)| \leq\left|k_{1} a_{0}-t k_{2} a_{1}\right| t+\left|\left(k_{2} a_{1}-t a_{2}\right)-\left(k_{2}-1\right) a_{1}\right| t^{2}+\sum_{j=3}^{\infty}\left|a_{j-1}-t a_{j}\right| t^{j} \leq \\
\leq t k_{1} a_{0}-t^{2} k_{2} a_{1}+t^{2} k_{2} a_{1}-t^{3} a_{2}+\left(k_{2}-1\right) t^{2} a_{1}+t^{3} a_{2}-t^{4} a_{3}+t^{4} a_{3}-t^{5} a_{4}+\cdots= \\
=t\left(k_{1} a_{0}+\left(k_{2}-1\right) t a_{1}\right) .
\end{array}
$$

Therefore, by the Schwarz Lemma,

$$
|\phi(z)| \leq\left(k_{1} a_{0}+\left(k_{2}-1\right) t a_{1}\right)|z| \quad \text { for } \quad|z| \leq t .
$$

Hence, for $|z| \leq t$

$$
|F(z)| \geq\left|t a_{0}+\left(\left(k_{1}-1\right) a_{0}-\left(k_{2}-1\right) t a_{1}\right) z\right|-\left|k_{1} a_{0}+\left(k_{2}-1\right) t a_{1}\right||z|>0
$$

if

$$
\left|\left(\left(k_{1}-1\right) a_{0}-\left(k_{2}-1\right) t a_{1}\right) z+t a_{0}\right|>\left|k_{1} a_{0}+\left(k_{2}-1\right) t a_{1}\right||z|,
$$

that is, $F(z)$ and therefore $f(z)$ does not vanish in

$$
\left(k_{1} a_{0}+\left(k_{2}-1\right) t a_{1}\right)|z|<\left|\left(\left(k_{1}-1\right) a_{0}-\left(k_{2}-1\right) t a_{1}\right) z+t a_{0}\right|,
$$

which is precisely the region

$$
\left|z-\frac{\left(k_{1}-1\right) a_{0} t-\left(k_{2}-1\right) a_{1} t^{2}}{\left(2 k_{1}-1\right)\left(a_{0}+2\left(k_{2}-1\right) a_{1} t\right)}\right|<\frac{k_{1} a_{0} t+\left(k_{2}-1\right) a_{1} t^{2}}{\left(2 k_{1}-1\right)\left(a_{0}+2\left(k_{2}-1\right) a_{1} t\right)} .
$$

That completes the proof of Theorem 2 .

\section{References}

1. Cauchy A. L. Exercises de mathématique. Oeuvres 9, 122 (1829).

2. Marden M. Geometry of Polynomials. In: Math. Surveys, no. 3. Amer. Math. Soc. Providence, RI (1966).

3. Milovanović G. V., Mitrinović D. S., Rassias Th. M. Topics in Polynomials: Extremal Problems, Inequalities, Zeros. World Scientific Publications (1994).

4. Joyal A., Labelle G., Rahman Q. I. On the Location of Zeros of Polynomials. Canadian Math. Bull. 10, 53-63 (1967). https://doi.org/10.4153/CMB-1967-006-3

5. Aziz A., Zargar B. A. Some Extensions of Enestrom - Kakeya Theorem. Glasnick Matematicki 31, 239-244 (1996). 

(2002).

6. Rahman Q. I., Schmeisser G. Analytic theory of Polynomials. Clarendon Press Oxford, 243-270

7. Aziz A., Shah W. M. On the location of zeros of polynomials and related analytic functions. Nonlinear Studies 6, 91-101 (1999).

Received: March 24, 2020

Revised: November 16, 2020

Accepted: December 17, 2020

Authors' information:

Nisar Ahmad Rather - Dr. Sci., Professor; dr.narather@gmail.com

Ishfaq Dar - PhD; ishfaq619@gmail.com

Aaqib Iqbal - itz.a.iqbal@gmail.com

\title{
Об областях, содержащих все нули полиномов, и связанных с ними аналитических функциях
}

\author{
Н. А. Ратхер, И. Дар, А. Икбал
}

Кашмирский университет, Индия, 190006, Сринагар

Для цитирования: Rather N.A., Dar I., Iqbal A. On the regions containing all the zeros of polynomials and related analytic functions // Вестник Санкт-Петербургского университета. Математика. Механика. Астрономия. 2021. Т.8(66). Вып. 2. С. 331-337. https://doi.org/10.21638/spbu01.2021.212

В этой статье с использованием стандартных методов получены результаты с ослабленной гипотезой, которые дают границы нулей для большего класса многочленов. Результаты авторов не только обобщают некоторые хорошо известные результаты, но также дают более точную информацию о местоположении нулей. Также получен аналогичный результат для аналитических функций. В дополнение к этому на примерах показано, что получена более точная информация о границах нулей полиномов, чем в некоторых известных работах.

Ключевые слова: многочлены, нули, комплексная область.

Статья поступила в редакцию 24 марта 2020 г.; после доработки 16 ноября 2020 г.; рекомендована в печать 17 декабря 2020 г.

Контактная информация:

Paтxep Hисар Aхмад - dr.narather@gmail.com

Дар Иифак - ishfaq619@gmail.com

Икбал Акиб - itz.a.iqbal@gmail.com 